\title{
Comparison of kernel density function in detecting effects of daily emission of Sulphur (IV) oxide from an industrial plant
}

\author{
Siloko Israel Uzuazor ${ }^{1,}$, Ejakpovi Simeon Uyovwieyovwe², Ukhurebor Kingsley \\ Eghonghon $^{3}$, Siloko Edith Akpevwe ${ }^{4}$, Ishiekwene Cyril Chukwuka ${ }^{4}$ \\ ${ }^{1}$ Department of Mathematics and Computer Science, Edo State University Uzairue, \\ Nigeria \\ ${ }^{2}$ Department of Mathematics, College of Education, Warri, Delta State, Nigeria \\ ${ }^{3}$ Department of Physics, Edo State University Uzairue, Nigeria \\ ${ }^{4}$ Department of Statistics, University of Benin, Nigeria
}

\begin{abstract}
Air pollution is a major concern of environmentalists because of the importance of air to man and other living organisms. This paper is about the investigation on the effects of daily emission of Sulphur (IV) oxide from an industrial pollutant using a nonparametric estimator which is the kernel estimator. Nonparametric estimators are free from distributional assumptions owing to the fact that most real-life data are not from a particular family of distribution. The functionality of this estimator is contingent on the smoothing parameter also called the bandwidth that determines the degree of the smoothness applied when analyzing the data. The bandwidth is extrapolated by minimizing the asymptotic mean integrated squared error which is the objective function of the kernel estimator. In this investigation, we selected some kernel functions of the beta family with the Gaussian kernel and obtained their bandwidths or smoothing parameters with respect to their distribution. The result of the analysis showed that an increase in number of tons of Sulphur (IV) oxide was associated with higher concentration level of the gas which suggests a potential danger of the gas to humans, animals and plants in the environment.
\end{abstract}

Keywords: Bandwidth; Kernel density estimation; Nonparametric; Sulphur (IV) oxide.

DOI: $\underline{\text { https://dx.doi.org/10.4314/ejst.v15i1.4 }}$

\section{INTRODUCTION}

Density estimation has been extensively employed in innumerable fields of studies in the examination of data with the aim of extracting relevant information from the

\footnotetext{
*Corresponding author: sisraelgreat@gmail.com, siloko.israel@edouniversity.edu.ng

(C) This is an Open Access article distributed under the terms of the Creative Commons Attribution License (http://creativecommons.org/licenses/CC BY4.0)
} 
data whose family of distribution is unknown conventionally. One of the essential nonparametric estimators in density estimation is the kernel estimator which uses the random variables in the production of probability estimates. The kernel estimator is a probability density function simply because it integrates to unity and hence it is a non-negative function. In practice, the kernel estimator comprises of a function and a coefficient of smoothness called the smoothing parameter, tuning parameter or bandwidth which regulates the smoothness level to be applied to the random variables. The popularity of the kernel estimator is accredited to the simplicity of its estimation procedure and interpretation of results. Irrespective of the indisputable applications of the estimator amongst many fields of human endeavors, its implementation is usually hampered by the selection process of the bandwidths with little emphasis on the kernel function owing to the fact that many kernel functions are probability density function (Siloko et al., 2020).

Nonetheless, the indispensable role of kernel method is more evident in recent statistical analysis in numerous disciplines (Denoel and Ficetola, 2015; Connor et al., 2019; Borrajo and Cao, 2020; Cortés-López and Jornet-Sanz, 2020). The usefulness of kernel density estimation has not been fully explored however an extension of its uses is found in signal processes, econometrics, chemo-informatics, optimization of molecular structure, geometrical molecular analysis and other gradient based optimization (McCabe et al., 2014). In deep neutral networks, the kernel method has been applied in investigating spectral distribution with the aid of the implicit kernel techniques where training and inferences were examined using Fourier features by sampling randomly (Li et al., 2019). Nonparametric estimation plays a major role in statistical analysis of data especially in structures identification where the observations are to "speak for themselves" with no stringent assumptions on the underlying probability distribution of the observations (Silverman, 2018). Nonparametric estimators unlike their parametric counterparts are flexible in implementation with no fixed structures while the parametric case has fixed structures with distributional assumption. One of the advantages of nonparametric estimation is that it considers all the data points in the estimation process and not just estimating some parameters of interest as typically practiced in parametric estimation. One of the popular nonparametric estimators is the kernel estimator which requires kernel function and smoothing parameter known as bandwidths or window width. Kernel density estimation involves the construction of a probability density estimates from a given sample with no assumptions about the probability distribution function of the observations (Siloko et al., 2018).

The aim of this study was to examine the effects of Sulphur (IV) oxide on the environment using the beta polynomial kernels with emphasis on the first six members of the family and the limiting case which is the Gaussian kernel function 
that is of numerous uses in data analytics. There are numerous industrial pollutants with adverse effects on humans, plants and animals but this paper considered the data of Sulphur (IV) oxide only.

\section{THEORETICAL FRAMEWORK OF KERNEL ESTIMATOR}

The kernel estimation method that was introduced by Rosenblatt (1956) and Parzen (1962) is one of the useful nonparametric estimators with its univariate form given as

$$
\hat{f}(t)=\frac{1}{n \lambda} \sum_{i=1}^{n} K\left(\frac{t-X_{i}}{\lambda}\right),
$$

where $K$ is kernel function, $n$ is sample size and $\lambda>0$ is the tuning parameter. Every kernel estimator is a probability density function and hence must satisfy the following presumptions

$$
\int K(t) d t=1, \int t K(t) d t=0 \text { and } \int t^{2} K(t) d t \neq 0 .
$$

The tuning parameter also called smoothing parameter or bandwidth that determines the degree of smoothness of the kernel estimate is very crucial in detecting the behavior of data (Wand and Jones, 1995; Chacón, 2009; Chacón and Duong, 2010; Zhang, 2015; Borrajo et al., 2017; Dhaker et al., 2018). Different techniques of obtaining the tuning parameter have been propounded by several authors and no single approach is universally accepted in all conditions, hence new tuning parameter selectors are being proposed. Recently, an automatic tuning parameter selector for recursive kernel estimators was developed by Slaoui (2019) for data which are length-biased while Davies and Lawson (2019) examined tuning parameter selectors that are based on the leave-one-out cross-validation algorithms in approximating likelihood functions mainly for spatial estimate. Researchers are still introducing novel tuning parameter selectors since its accurate selection is of prime importance in kernel density estimation especially with increase in dimension (Varet et al., 2019; Bedouhene and Zougab, 2020; Tenreiro, 2020; Tsuruta and Sagae, 2020). If the kernel function is continuously differentiable, then the derivative of Equation (1) which is called kernel density derivative estimator is

$$
\hat{f}^{(m)}(t)=\frac{1}{n \lambda^{m+1}} \sum_{i=1}^{n} K^{(m)}\left(\frac{t-X_{i}}{\lambda}\right),
$$

where $K^{(m)}$ is the $m t h$ derivative of the kernel which is a symmetric probability density function and also satisfy the axioms of kernel function. Again, as in the case of the kernel density method, the smoothing parameter for kernel derivative must be appropriately chosen owing to the fact that larger values of the smoothing parameter 
are required for the implementation of kernel derivative and every new derivative order attracts two extra powers of the smoothing parameter in their estimation (Scott, 2015; Siloko et al., 2019a).

\section{OPTIMIZATION CRITERION FUNCTION OF KERNEL ESTIMATOR}

There is no generally acceptable method in estimating the "optimal" smoothing parameter in kernel estimation; nonetheless optimality in kernel method is usually with respect to some known optimization criteria functions. There are several model diagnostics and model performance measures in kernel estimation such as integrated absolute error and Kullback-Liebler distance that is the likelihood criterion. However, these criteria functions lack the dimensionality property when considering the multivariate setting of kernel estimation. The dimension of a kernel function provides numerous potential gains in application. One of the popular and most tractable performance measures or optimality functions in kernel estimation is the asymptotic mean integrated squared error whose result is dependent on the tuning parameter. The popularity of the asymptotic mean integrated squared error is due to its straight forward mathematical formulation unlike other error criteria functions with complex mathematical formulation. The asymptotic mean integrated squared error is made up of two components which are the integrated squared bias and the integrated variance given as

$$
\operatorname{AMISE}(\hat{f}(t))=\frac{R(K)}{n \lambda}+\frac{\lambda^{4}}{4} \mu_{2}(K)^{2} R\left(f^{\prime \prime}\right),
$$

where $R(K)=\int K(t)^{2} d t$ is the kernel roughness while $R\left(f^{\prime \prime}\right)=\int f^{\prime \prime}(\mathrm{x})^{2} d \mathrm{x}$ is the curvature or roughness of the unknown probability distribution function. There is the usual "bias-variance" trade-off because the bias and variance components of the AMISE depend on the tuning parameter. The tuning parameter with the least AMISE value is obtain by the minimization of Equation (4) with respect to $\lambda$ and is given as

$$
\lambda_{\text {AMISE }}=\left\{\frac{R(K)}{\mu_{2}(K)^{2} R\left(f^{\prime \prime}\right)}\right\}^{1 / 5} \times n^{-1 / 5} .
$$

Also, the corresponding AMISE of the $m t h$ derivative of the kernel function if $K$ is sufficiently differentiable is

$$
\operatorname{AMISE}\left(\hat{f}^{(m)}(t)\right)=\frac{R\left(K^{(m)}\right)}{n \lambda^{2 m+1}}+\frac{1}{4} \lambda^{4} \mu_{2}(K)^{2} R\left(f^{(m+2)}\right),
$$

where $R\left(K^{(m)}\right)$ is roughness of the $m t h$ derivative of the kernel, $\mu_{2}(K)^{2}$ is the second moment of the kernel and $R\left(f^{(m+2)}\right)$ is roughness of the $m t h$ unknown probability density function. As observed in Equation (5), the $m t h$ integrated variance of $\hat{f}^{(m)}(t)$ is $R\left(K^{(m)}\right) / n \lambda^{2 m+1}$ while the integrated squared bias of 
$\hat{f}^{(m)}(t)$ is $\frac{1}{4} \lambda^{4} \mu_{2}(K)^{2} R\left(f^{(m+2)}\right)$ with regards to kernel density derivatives estimation (Siloko et al., 2019b). The bias component of the $m t h$ derivative order is $O\left(\lambda^{4}\right)$ while the variance attracts two extra powers for the tuning parameter with new derivative order. The AMISE in Equation (6) will produce its minimum value when the tuning parameter is

$$
\lambda_{A M I S E}^{m}=\left[\frac{(2 m+1) R\left(K^{(m)}\right)}{\mu_{2}(K)^{2} R\left(f^{(m+2)}\right)}\right]^{\left(\frac{1}{2 m+5}\right)} \times n^{-\left(\frac{1}{2 m+5}\right)} .
$$

The tuning parameter required for kernel density derivative estimation is usually larger than kernel density estimation and therefore should be critically selected especially with increase in the derivatives orders and dimensions (Siloko et al., 2019c). Kernel derivative estimation should be consistent; that is the function must be continuously differentiable with larger tuning parameters for its effective implementation (Scott, 2015; Ejakpovi et al., 2019).

\section{CHOICE OF KERNEL FUNCTION}

Kernel estimation usually attracts two major questions which are choice of the kernel function and choice of the tuning parameter. The choice of the tuning parameter is more important in the estimation process because most kernel functions are probability density function. There are numerous kernel estimators with different applications primarily for exploration and visualization of data and more novel estimators were been propounded (Bouezmarni et al., 2020; Mugdadi and Sani, 2020; Harfouche et al., 2020; Bolancé and Acuña, 2021; Mohammed and Jassim, 2021). The general form of the beta polynomial kernel family is

$$
K_{[p]}(t)=\frac{(2 p+1) !}{2^{2 p+1}(p !)^{2}}\left(1-t^{2}\right)^{p},
$$

where $p=0,1,2, \ldots, \infty$ is the power of the polynomial (Hansen, 2005). This family of kernels are supported and evaluated within the interval $[-1,1]$. As $p$ takes values from 0 to 3, the resulting kernels are Uniform, Epanechnikov, Biweight and Triweight kernels. Amongst this kernel family, the simplest kernel is the uniform kernel while the normal kernel is the limiting case, which is when, $p$ goes to infinity. These classes of kernels are very useful especially in exploratory and visualization of features in data analysis. The investigation will be on the first six members of this family and the Gaussian kernel which is the limiting case while the uniform kernel known as the simplest kernel is excluded. The exclusion of the uniform kernel function is due to the fact that the second derivative of the kernel function does not exist; hence its bandwidth cannot be computed. The computation of the bandwidth requires the second derivative of the kernel function and this unique property is absent in the uniform kernel because it is not continuously differentiable. The 
bandwidth of the uniform kernel may be difficult to compute but other statistical properties such as its efficiency could be computed because the computation of the efficiency of kernel does not require the second derivative. The members of the beta family when $p=1,2,3,4,5,6$ are Epanechnikov, Biweight, Triweight, Quadriweight, Pentaweight and Hexaweight kernels which are as follows

$$
\begin{aligned}
K_{1}(t) & =\frac{3}{4}\left(1-t^{2}\right) . \\
K_{2}(t) & =\frac{15}{16}\left(1-t^{2}\right)^{2} . \\
K_{3}(t) & =\frac{35}{32}\left(1-t^{2}\right)^{3} . \\
K_{4}(t) & =\frac{315}{256}\left(1-t^{2}\right)^{4} . \\
K_{5}(t) & =\frac{693}{512}\left(1-t^{2}\right)^{5} . \\
K_{6}(t) & =\frac{3003}{2048}\left(1-t^{2}\right)^{6}
\end{aligned}
$$

However, when $p$ goes to infinity, the kernel obtained is the Gaussian kernel which is

$$
K_{\varnothing}(t)=\frac{1}{\sqrt{2 \pi}} \exp \left(-\frac{t^{2}}{2}\right) .
$$

The given kernel functions are usually regarded as second order kernels whose higher order forms can be obtained by the multiplicative or additive techniques. The higher order forms are regarded as bias reducing kernels as a result of their fast convergence rates (Marron, 1994).

\section{RESULTS AND DISCUSSION}

The kernel estimator is used in the visualization of daily emission of Sulphur (IV) oxide, $\mathrm{SO}_{2}$ in tons from an industrial plant as presented in Table 1 (Datta, 2013). Table 1 is the daily emission of Sulphur (IV) oxides in tons from an industrial plant arranged in increasing order of magnitude. Sulphur (IV) oxide is a noxious gas released from the oxidization of fossil materials which have adverse effect on man and its surroundings. The gas is heavier than air with strangulating smell in aerial concentration of 500 parts per billion (ppb) which depicts high level of fatality of the gas to man. At certain concentration levels of the gas, breathing problems, eye infuriation, chest pains, lung and heart related problems might occur (Wright, 2000). The concentration levels of $20 \mathrm{ppb}$ or below will have no effects on humans but the normal concentration levels of the gas in the atmosphere should be $10 \mathrm{ppb}$ or lower (Schnelle and Brown, 2002). As a result of the importance of the knowledge of 
Sulphur (IV) oxide, analyzing data that are related to the gas statistically is very important.

Table 1. Daily emission of Sulphur (IV) oxides in tons from an Industrial Plant.

\begin{tabular}{llllllllll}
\hline 6.20 & 7.70 & 8.30 & 9.40 & 9.80 & 9.90 & 10.5 & 10.7 & 11.0 & 11.2 \\
11.8 & 12.3 & 12.8 & 13.2 & 13.3 & 13.5 & 13.9 & 14.4 & 14.5 & 14.7 \\
15.2 & 15.5 & 15.8 & 15.9 & 16.2 & 16.7 & 16.9 & 17.0 & 17.3 & 17.5 \\
17.6 & 17.9 & 18.0 & 18.0 & 18.1 & 18.1 & 18.4 & 18.5 & 18.7 & 19.0 \\
19.1 & 19.2 & 19.3 & 19.4 & 19.4 & 20.0 & 20.1 & 20.1 & 20.4 & 20.5 \\
20.8 & 20.9 & 21.4 & 21.6 & 21.9 & 22.3 & 22.5 & 22.7 & 22.7 & 22.9 \\
23.0 & 23.5 & 23.7 & 23.9 & 24.1 & 24.3 & 24.6 & 24.6 & 24.8 & 25.7 \\
25.9 & 26.1 & 26.4 & 26.6 & 26.8 & 27.5 & 28.5 & 28.6 & 29.6 & 31.8 \\
\hline
\end{tabular}

The kernel estimates of the Sulphur (IV) oxides data using the selected kernels besides the Gaussian kernel indicated that the data were multimodal and the modes were located between 10 tons and 25 tons of the gas with the peak at 19 tons. The different peaks represented the concentration levels of the gas that are harmless and harmful to humans, animals, and plants as well. The probabilities of the peak of the estimates of the various kernel functions lie between 0.025 and 0.06 .

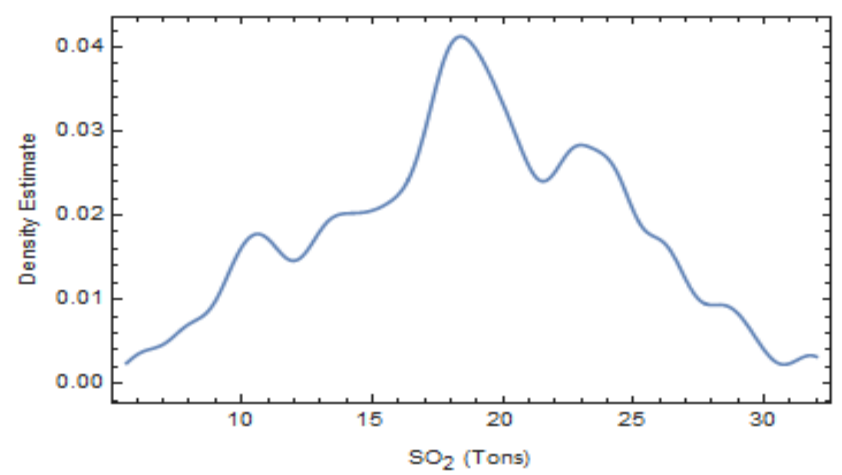

Figure 1. Epanechnikov kernel estimate showing tons of Sulphur (IV) oxides. 


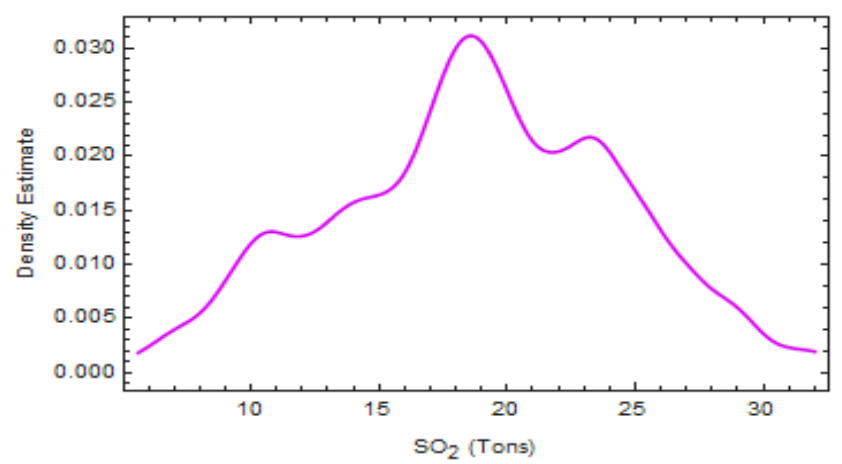

Figure 2. Biweight kernel estimate showing tons of Sulphur (IV) oxides.

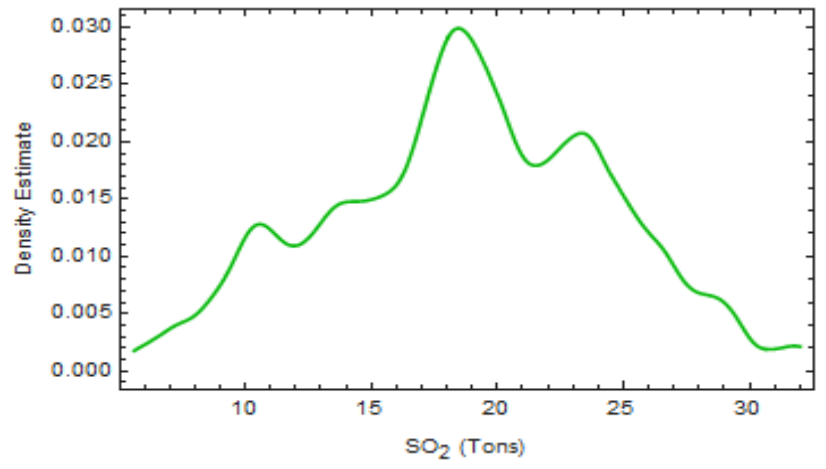

Figure 3. Triweight kernel estimate showing tons of Sulphur (IV) oxides.

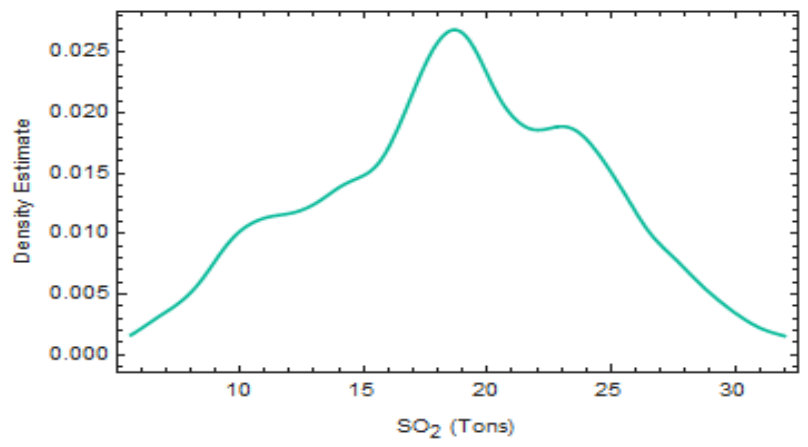

Figure 4. Quadriweight kernel estimate showing tons of Sulphur (IV) oxides. 


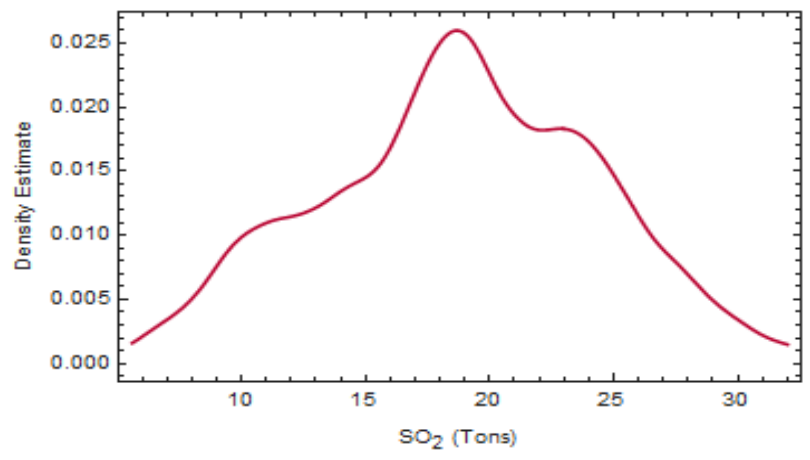

Figure 5. Pentaweight kernel estimate showing tons of Sulphur (IV) oxides.

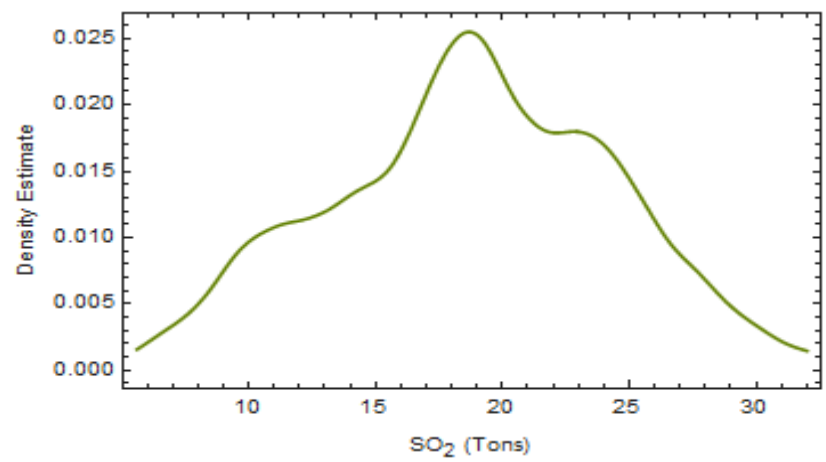

Figure 6. Hexaweight kernel estimate showing tons of Sulphur (IV) oxides.

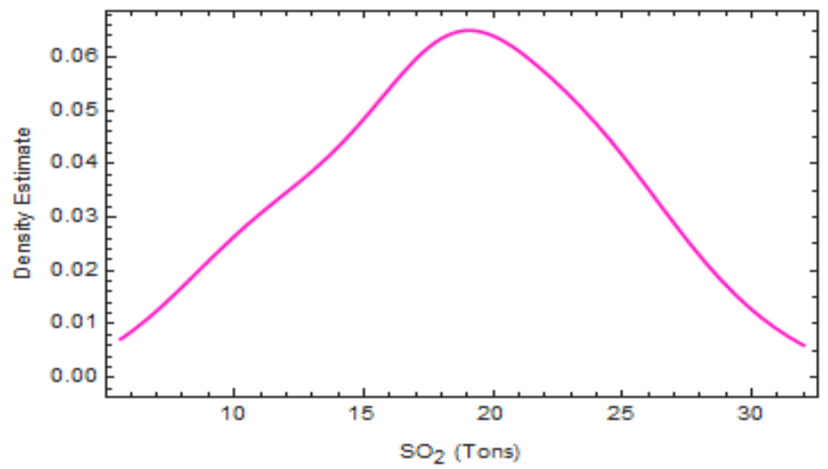

Figure 7. Normal kernel estimate showing tons of Sulphur (IV) oxides. 
Sulphur (IV) oxide is a colorless and reactive air pollutant with a strong odor. The gas is mainly obtained from fossil fuel combustions and volcanic activities. High levels of concentration of Sulphur (IV) oxide usually cause respiratory related diseases such as inflammation and irritation of the respiratory system. Despite these negative effects of Sulphur (IV) oxide at high concentration levels, the gas is still of valuable applications in a control situation. One of the most important applications of Sulphur (IV) oxide is the preparation of Sulphuric acid which is widely used in industries. Apart from the production of Sulphuric acid, the gas can also be used as disinfectant, refrigerant, reducing agent, bleach, and food preservative particularly for dried fruits (Liu et al., 2020).

The kernel estimates of the Sulphur (IV) oxide data clearly indicated regions where the gas can be dangerous to humans and its environment. The peaks of the kernel estimates (graphs) are 19 tons which simply means that the gas is harmless between the least value of 6.2 tons and 19 tons with respect to the level of concentration that is emitted within that range. The concentration levels of the gas below $20 \mathrm{ppb}$ does not affect humans and the environment negatively and from the kernel estimates, the number of tons between 6 tons and 19 tons emitted daily from the industrial plant will have no adverse effects on the environment. At number of tons of the gas that are above 19 tons due to the activities of the industry, the required level of $20 \mathrm{ppb}$ will be exceeded and the negative effects of the gas will be experienced by humans, animals and even plants. The normal concentration levels of Sulphur (IV) oxide in the atmosphere is $10 \mathrm{ppb}$ and below and this is obviously displayed in the kernel estimates of Epanechnikov, Biweight, and Triweight and also noticed in the other studied kernels apart from the Normal kernel with smooth estimate. The point of normalcy of the gas is the first mode (bump) as the graphs rises before it gets to its peak and the value of that bump is within the emission region of 10 tons. At emission level above 20 tons daily, the graphs start falling which is an indication that humans, plants and animals will be drastically affected since the volume of the gas released into the atmosphere is beyond the prescribed level of concentrations required for normal living. Again, it should be noted that as the power of the polynomial increases, the kernel estimates produced are smoother but this is also capable of smoothing away important informative features of the data.

As descried by the Normal kernel estimate in Figure 7, the data examined displayed unimodality contrary to the kernel estimates of Epanechnikov, Biweight, and the other studied kernels where the data exhibited multimodality. The exhibition of unimodality by the Normal kernel is occasioned by the magnitude of the bandwidth together with the continuous differentiability property and hence the production of a smoother estimates unlike members of the beta polynomial kernels. The peak of the Normal kernel estimate which is the mode is at 19 tons in consonance with the 
other kernel estimates. Generally, the smoothness of kernel estimates is fundamentally depended on the smoothing parameter which determines and also regulates the contributions of the bias and variance components to the AMISE value. Table 2 is the statistical properties and performance measures of Sulphur (IV) oxides data with the AMISE as the error criterion function of the selected kernels. The results in Table 2 show that the Epanechnikov kernel produced the least value of the AMISE amongst the beta kernels and that supports its optimality property with respect to the AMISE as an error criterion function. This can also be viewed that amidst the beta kernel family, in terms of risk detection of the industrial pollutant, the Epanechnikov kernel can easily detect the slightest changes in the environment regarding the volume of Sulphur (IV) oxide released into the atmosphere.

Table 2. Statistical properties and risk measures of industrial pollution effects.

\begin{tabular}{|c|c|c|c|c|c|c|c|}
\hline Kernel Functions & $\boldsymbol{R}(\boldsymbol{K})$ & $\mu_{2}(K)$ & $R\left(f^{\prime \prime}\right)$ & $\lambda$ & Variance & Bias $^{2}$ & AMISE \\
\hline$K_{1}(t)=\frac{3}{4}\left(1-t^{2}\right)$ & $\frac{3}{5}$ & $\frac{1}{5}$ & $\frac{9}{2}$ & 1.0453 & 0.0451 & 0.0023 & 0.0473 \\
\hline$K_{2}(t)=\frac{15}{16}\left(1-t^{2}\right)^{2}$ & $\frac{5}{7}$ & $\frac{1}{7}$ & $\frac{45}{2}$ & 1.2499 & 0.0922 & 0.0024 & 0.0945 \\
\hline$K_{3}(t)=\frac{35}{32}\left(1-t^{2}\right)^{3}$ & $\frac{350}{429}$ & $\frac{1}{9}$ & 35 & 1.3128 & 0.1122 & 0.0017 & 0.1139 \\
\hline$K_{4}(t)=\frac{315}{256}\left(1-t^{2}\right)^{4}$ & $\frac{2204}{2431}$ & $\frac{1}{11}$ & $\frac{8505}{143}$ & 1.3925 & 0.1420 & 0.0015 & 0.1435 \\
\hline$K_{5}(t)=\frac{693}{512}\left(1-t^{2}\right)^{5}$ & $\frac{4158}{4199}$ & $\frac{1}{13}$ & $\frac{20790}{221}$ & 1.4653 & 0.1741 & 0.0013 & 0.1754 \\
\hline$K_{6}(t)=\frac{3003}{2048}\left(1-t^{2}\right)^{6}$ & $\frac{26679}{25000}$ & $\frac{1}{15}$ & $\frac{45045}{323}$ & 1.5308 & 0.1754 & 0.0012 & 0.1765 \\
\hline$K_{\varnothing}(t)=\frac{1}{\sqrt{2 \pi}} \exp \left(-\frac{t^{2}}{2}\right)$ & $\frac{1}{2 \sqrt{\pi}}$ & 1 & $\frac{3}{8 \sqrt{\pi}}$ & 2.4716 & 0.0014 & 0.0004 & 0.0018 \\
\hline
\end{tabular}

Despite the production of the least AMISE value amongst the beta kernel functions, the estimate of the Epanechnikov kernel is wiggly in contrast with the estimate of the Normal kernel. The wiggly Epanechnikov kernel estimate and smooth Normal kernel estimate are mainly attributed to the magnitude of the bandwidth as presented in Table 2. The Gaussian kernel produced the largest smoothing parameter which resulted in the production of a smooth estimate and also with the smallest AMISE 
value in all the studied functions. Although the Epanechnikov kernel is the optimal kernel of the beta polynomial family with regards to the AMISE, oftentimes inaccurate statistical information about the observations been investigated may be divulged by the estimate. The prominence of the Normal kernel in density estimation and data analysis is on the basis of its production of smooth density estimates that are devoid of erroneous findings. The strength of the kernel function in detection of risk in the environment decreases as the power of the polynomial kernel increases. The estimates of the kernel functions studied are similar and the similarity is a clear indication that higher probabilities values are associated with higher tons of the Sulphur (IV) oxide. The tuning parameter which regulates the contribution of the components of the AMISE increases as the power of the kernel increases and this accounted for the increase in the AMISE value which is regarded as the risk measure. However; with the Gaussian kernel which is strictly not a member of the beta kernel family, the AMISE value reduces drastically despite the increase of the bandwidth implying that the Normal kernel surmounts the other kernel functions explored.

\section{CONCLUSION}

This paper discusses the potentials of kernel estimators in detecting the effects of the volume of Sulphur (IV) oxide released into the atmosphere daily from an industrial plant. The rising level of the effects of the gas is displayed in the kernel estimates and the regions of high number of tons of the gas with adverse effects in the environment are vividly shown. When the number of tons emitted daily is above 20 tons, the prescribed concentration levels of the gas in the atmosphere will be exceeded which resulted in increase in probabilities values. The increase in probabilities values associated with increase in the number of tons emitted daily is an indication that human lives, plants and animals shall experience the negative effects of the gas in the environment. Hence; appropriate government authority should enact laws that should regulate the emission of Sulphur (IV) oxide into the environment.

\section{ACKNOWLEDGEMENTS}

The authors appreciate the anonymous reviewers for painstakingly going through the manuscript and for their valuable comments that have improved the manuscript.

\section{Competing interests}

The authors declared that they have no conflict of interest. 


\section{REFERENCES}

Bedouhene, K and Zougab, N. (2020). A Bayesian procedure for bandwidth selection in circular kernel density estimation. Monte Carlo Methods and Applications 26(1): 69-82.

Bolancé, C and Acuña, C. A. (2021). A new kernel estimator of copulas based on beta quantile transformations. Mathematics 9: 1078.

Borrajo, L and Cao, R. (2020). Big-But-Biased data analysis for air quality. Electronics 9.

Borrajo, M. I., González-Manteiga, W and Martìnez-Miranda, M. D. (2017). Bandwidth selection for kernel density estimation with length-biased data. Journal of Nonparametric Statistics 29: 636-668.

Bouezmarni, T., Bellengem, S. and Rabbi, Y. (2020). Nonparametric beta kernel estimator for long and short memory time series. The Canadian Journal of Statistics 48: 582-595.

Chacón, J. E. (2009). Data-driven choice of the smoothing parametrization for kernel density estimators. The Canadian Journal of Statistics 37: 249-265.

Chacón, J. E. and Duong, T. (2010). Multivariate plug-in bandwidth selection with unconstrained pilot bandwidth matrices. Test 19: 375-398.

Connor, C. B., Connor, L. J., Germa, A., Richardson, J. A., Bebbington, M., Gallant, E. and Saballos, J. A. (2019). How to use kernel density estimation as a diagnostic and forecasting tool for distributed volcanic vents. Statistics in Volcanology 4(3): 1-25.

Cortés López, J. C. and Jornet-Sanz, M. (2020). Improving kernel methods for density estimation in random differential equations problems. Mathematical and Computational Applications 25(2): 33. doi: $10.3390 / \mathrm{mca} 25020033$

Davies, T. M. and Lawson A. B. (2019). An evaluation of likelihood-based bandwidth selectors for spatial and spatiotemporal kernel estimates. Journal of Statistical Computation and Simulation 89(7): $1131-1152$.

Datta, K. B. (2013). Mathematical methods of science and engineering. Cengage Learning, India.

Denoel, M. and Ficetola, G. F. (2015). Using kernels and ecological niche modeling to delineate conservation areas in an endangered patch-breeding phenotype. Ecological Applications 25(7): 1922-1931.

Dhaker, H., Ngom, P., Deme, E-H, and Mbodj, A. (2018). New approach for bandwidth selection in the kernel density estimation based on $\beta$-divergence. Journal of Mathematical Sciences: Advances and Applications 51: 57-83.

Ejakpovi, S. U., Ozobokeme, J. K and Uveruveh, F. O. (2019). Generalized efficiency of kernel density derivative estimation: A univariate perspective. Journal of Mathematical Association of Nigeria 46(1): 460-464

Hansen, B. E. (2005). Exact mean integrated squared error of higher order kernel estimators. Econometric Theory 21: 1031-1057.

Harfouche, L., Zougab, N. and Adjabi, S (2020). Multivariate generalized gamma kernel density estimators and application to non-negative data. International Journal of Computing Science and Mathematics, 11(2): 137-157.

Li, C-L., Chang, W-C., Mroueh, Y., Yang, Y. and Póczos, B. (2019). Implicit kernel learning. Proceedings of the $22^{\text {nd }}$ International Conference on Artificial Intelligence and Statistics (AISTATS), Naha, Okinawa, Japan. PMLR: Volume 89.

Liu, T., Clegg, S. L., and Abbatt, J. P. D. (2020). Fast oxidation of sulfur dioxide by hydrogen peroxide in deliquesced aerosol particles. Proceedings of the National Academy of Sciences of the United States of America 117(3): 1354-1359.

Marron, J. S. (1994). Visual understanding of higher-order kernels. Journal of Computational and Graphical Statistics 3(4): 447-458.

McCabe, P., Korb, O. and Cole, J. (2014). Kernel density estimation applied to bond length, bond angle, and torsion angle distributions. Journal of Chemical Information and Modeling 54(5): 1284-1288. 
Mohammed, A. D. and Jassim, N. H. (2021). A comparison study of nonparametric kernel estimators with gaussian weight function. Journal of Physics: Conference Series, 1818012058.

Mugdadi, A. and Sani, R. (2020). The Linear Combination of Kernels in the Estimation of Cumulative Distribution Functions. Applications and Applied Mathematics, 15(2): 875-893.

Parzen, E. (1962). On the estimation of a probability density function and the mode. Annals of Mathematical Statistics 33: 1065-1076.

Rosenblatt, M. (1956). Remarks on some nonparametric estimates of a density function. Annals of Mathematical Sciences 27: 832-837.

Schnelle, K.B and Brown, C.A. (2002). Air pollution control technology handbook. Publisher CRC Press.

Scott, D.W. (2015). Multivariate density estimation. Theory, practice and visualisation. Second Edition, Wiley, New Jersey.

Siloko, I.U., Ishiekwene, C.C. and Oyegue, F.O. (2018). New gradient methods for bandwidth selection in bivariate kernel density estimation. Journal of Mathematics and Statistics 6(1): 1-8.

Siloko, I. U., Siloko, E. A., Ikpotokin, O., Ishiekwene, C. C. and Afere, B. A. (2019a). On asymptotic mean integrated squared error's reduction techniques in kernel density estimation. International Journal of Computational and Theoretical Statistics 6(1): 89-98.

Siloko, I. U., Ikpotokin, O., Oyegue, F. O., Ishiekwene, C. C. and Afere, B. A. (2019b). A note on application of kernel derivatives in density estimation with the univariate case, Journal of Statistics and Management Systems 22(3): 415-423.

Siloko, I. U., Ikpotokin, O. and Siloko, E. A. (2019c). A key note on performance of smoothing parameterizations in kernel density estimation. Tanzania Journal of Science 45(1): 1-8.

Siloko, I. U., Nwankwo, W. and Siloko, E. A. (2020). A new family of kernels from the beta polynomial kernels with applications in density estimation. International Journal of Advances in Intelligent Informatics 6(3): 235-245

Silverman, B. W. (2018). Density estimation for statistics and data analysis. Routledge, New York.

Slaoui, Y. (2019). Automatic bandwidth selection for recursive kernel density estimators with lengthbiased data. Japanese Journal of Statistics and Data Science. https://doi.org/10.1007/s42081-019$\underline{00053-\mathrm{Z}}$

Tenreiro, C. (2020). Bandwidth selection for kernel density estimation: A Hermite series-based direct plug-in approach. Journal of Statistical Computation and Simulation 90(18): 3433-3453

Tsuruta, Y. and Sagae, M. (2020). Theoretical properties of bandwidth selectors for kernel density estimation on the circle. Annals of the Institute of Statistical Mathematics 72: 511-530.

Varet, S., Lacour, C., Massart, P. and Rivoirard, V. (2019). Numerical performance of penalized comparison to overfitting for multivariate kernel density estimation. https://hal.inria.fr/hal$\underline{02002275 /}$

Wand, M. P. and Jones, M. C. (1995). Kernel smoothing. Chapman and Hall, London.

Zhang, J. (2015). Generalized least squares cross-validation in kernel density estimation. Statistica Neerlandica 69(3): 315-328.

Wright, A. A. (2000). Exhaust emissions from combustion machinery. Marine Engineering Practice Series 3(20). 